\title{
Aberrant neural crest development causes craniofacial and other malformations in an animal model of Musculocontractural Ehlers-Danlos syndrome
}

\author{
Edgar M. Pera ${ }^{1 *}$, Nadège Gouignard' and Marco Maccarana ${ }^{2}$ \\ 'Lund Stem Cell Center, Lund University, 22184 Lund, Sweden \\ ${ }^{2}$ Dept. Experimental Medical Science, Lund University, 22184 Lund, Sweden
}

Article Info

\section{Article Notes}

Received: October23, 2016

Accepted: November 28, 2016

\section{${ }^{*}$ Correspondence:}

Dr. Edgar M. Pera, Lund Stem Cell Center, BMC, D10 Klinikgatan 32, SE-221 84 Lund, Sweden, Tel. (46) 46 2221738, E-mail: edgar.pera@med.lu.se

C 2016 Edgar M. Pera. This article is distributed under the terms of the Creative Commons Attribution 4.0 International License.

\section{Keywords}

Musculocontractural Ehlers-Danlos syndrome

Neural crest

Craniofacial development

Proteoglycan

Dermatan sulfate

\section{ABSTRACT}

Musculocontractural Ehlers-Danlos syndrome (MC-EDS) is a rare recessive disorder that is characterized by connective tissue fragility, distinct craniofacial features and congenital malformations. MC-EDS patients have defects in the enzymes dermatan sulfate epimerase-1 and dermatan 4-0-sulfotransferase-1, which are involved in the biosynthesis of iduronic acid in the chondroitin sulfate/dermatan sulfate (CS/DS) chains of proteoglycans (PGs). While the connective tissue defect is a result of disturbed collagen fibril assembly based on a decreased iduronic acid content of interacting CS/DS-PGs, the cause of the developmental malformations in MC-EDS is not well understood. This review focuses on a new role of CS/DS-PGs in the development of multipotent and highly migratory neural crest (NC) cells in the Xenopus embryo model of MCEDS. Single iduronic acid residues in CS/DS-PGs are involved in the formation of NC-derived craniofacial structures by facilitating the migration and adhesion of NC cells to fibronectin. Our results suggest a defect in NC development as cause of the craniofacial and other congenital anomalies in MC-EDS patients, which might contribute to an improved diagnosis and etiology-based therapy.

\section{Text}

The Ehlers-Danlos syndrome (EDS) is a heterogeneous group of rare monogenetic diseases that is characterized by connective tissue fragility and defects in skin, ligaments, joints, blood vessels, and internal organs ${ }^{1}$. General muscle weakness and joint contractures are eponymous to the newly described musculocontractural (MC) subtype of EDS $^{2,3}$. Commonly encountered in MC-EDS are also developmental anomalies and a set of craniofacial dysmorphic features, such as hypoplasia of the jaw, high and/or cleft palate, midface skeletal anomaly, downslanting palpedral fissures and malformed ears. MC-EDS is a recessive disorder and caused by homozygous loss-of-function mutations in two genes, which are cooperating in the biosynthesis of chondroitin/dermatan sulfate, i.e. CHST14 encoding dermatan 4-0-sulfotransferase-1 (D4ST1; 31 patients reported) and DSE encoding dermatan sulfate epimerase-1 (DS-epi1; 3 patients reported) 3,4,5. $^{3}$.

Chondroitin sulfate/dermatan sulfate proteoglycans (CS/ DS-PGs) are abundant components on the cell surface and in the extracellular matrix (ECM) of connective tissues. They are involved in a wide spectrum of biological and pathological processes, including ECM organization, growth factor signaling, proliferation, survival, adhesion, migration, coagulation, bacteria attachment, 
and atherosclerotic plaque formation ${ }^{6,7,8}$. Usually both the core protein and the attached glycosaminoglycan (GAGs) chains determine the biological function of a PG. GAGs are long chains of repeating disaccharide subunits that are covalently bound to a variety of PGs. A tetrasaccharide linker (glucuronic acid-galactose-galactose-xylose-core protein) is common to CS/DS and heparan sulfate. The addition of $\mathrm{N}$-acetylgalactosamine to this linker sequence distinguishes CS/DS from heparan sulfate. DS is formed from CS in two enzymatic steps. DS-epi1 first epimerizes a C5 carboxyl group, which transforms glucuronic acid, present in CS, to iduronic acid (IdoA), present in DS. This conversion is not quantitative, and therefore the final hybrid chain is called CS/DS. D4ST1 then catalyzes 4-O-sulfation of $\mathrm{N}$-acetylgalactosamine, which prevents back-epimerization of the adjacent IdoA.

Several variants of EDS are caused by mutations in enzymes that affect the biosynthesis of $\mathrm{PGs}^{1}$. In patients with the very rare progeroid form of $\operatorname{EDS}^{9}$ and a pleiotropic EDS-like disorder ${ }^{10,11}$, hypomorphic homozygous mutations in galactosyltransferase 1 and galactosyltransferase 2 , respectively, reduce the ability to synthesize the tetrasaccharide linker sequences and therefore the whole GAGs. MC-EDS is the first human disorder that specifically affects the CS/DS biosynthesis ${ }^{2}$. The reported missense mutations R267G and S268L decrease the activity of DS-epi1 and consequently lead to a reduced IdoA fraction in CS/DSPGs, such as decorin ${ }^{4,5}$. Studies of the skin in Dse knockout mice $^{13}$ and cultured fibroblasts from D4ST1-deficient human patients ${ }^{5}$ revealed that the dermal connective tissue fragility results from impaired assembly of collagen fibrils due to an altered composition in the decorin CS/DS chain. Some Dse knockout mice in pure genetic background have abdominal wall and neural tube closure defects during fetal development ${ }^{14}$. In addition, keratinocyte differentiation is altered. No craniofacial dysmorphic features have been reported in the mouse model but are present in patients. Etiological and molecular explanations for these MC-EDS defects have not been put forward yet.

We noted that several craniofacial abnormalities in MC-EDS are also observed in Treacher Collins syndrome, Nager syndrome, and Miller syndrome ${ }^{15}$. These congenital disorders are associated with defects in an embryonic multipotent cell population, designated the neural crest (NC). The NC arises at the border between the neural and epidermal ectoderm in the vertebrate embryo ${ }^{16,17}$. NC cells undergo epithelial-to-mesenchymal transition (EMT), migrate along restricted pathways, and contribute to nearly every organ system in the body, including the craniofacial skeleton, melanocytes, and the peripheral nervous system. Abnormal NC development leads to a diverse group of pathologies known as neurocristopathies ${ }^{18}$.
Studies in amphibian, avian and mouse embryos revealed that the CS-PGs aggrecan and versicans V0 and V1 act in the ECM as guidance cues for NC cell migration ${ }^{19,20,21}$. Aggrecan is an inhibitory cue, and the versicans have been presented as both positive and negative regulators of NC cell migration. In Xenopus, versicans are expressed in tissues surrounding the migratory cranial neural crest (CNC) and enhance directional migration by confining the pathway of NC cells ${ }^{22,23}$. However, CS/DS-PGs that are expressed by the migratory CNC cells and the functions of IdoA in NC cell migration have not been investigated yet.

Due to their high number, large size and external development, embryos of the African claw frog Xenopus laevis constitute a favorable experimental model. The high degree of gene conservation makes this model amenable for the study of human diseases. We identified a defect in $\mathrm{NC}$ development as a possible underlying cause for craniofacial abnormalities and other congenital manifestations in MC-EDS ${ }^{24}$. In the Xenopus embryo, the Dse gene was expressed in the epidermal ectoderm, premigratory NC and migrating CNC cells. The inhibition of DS-epi1 activity by microinjection of antisense morpholino oligonucleotides did not affect the allocation of neural and epidermal fates nor the formation of early NC progenitors. However, impaired EMT and reduced extent of NC cell migration was observed, leading to a decrease in NCderived melanocytes, dorsal fin and craniofacial skeleton structures. This effect was specific, because injection of a human DS-epi1 expression plasmid restored normal NC migration in morphant embryos, whereas a catalytically inactive mutant DS-epi1 (H205A) construct ${ }^{25}$ did not have any effect $^{24}$. Using transplantation of green fluorescent protein-labelled CNC explants, we demonstrate that DSepi1 deficiency in the CNC but not in adjacent tissue impaired CNC cell migration. The culture of CNC explants revealed that DS-epi1 is important for cell spreading and the formation of lamellipodia and filopodia on fibronectin. Single cell cultures further uncovered a role of IdoA in the adhesion of CNC cells to this matrix protein. In Xenopus, fibronectin flanks the NC cell migration pathways in the embryo $^{26}$ and is the only ECM substrate tested so far that supports CNC migration in vitro ${ }^{27}$. Purified CS/DS binds to fibronectin ${ }^{28}$. Abrogation of DS-epi1 by short hairpin RNA-mediated gene silencing in a human esophagus squamous cell carcinoma cell line ${ }^{29}$ and in cultured aortic smooth muscle cells from null-mutant mice ${ }^{30}$ causes reduced plasma membrane protrusions and abnormal actin cytoskeleton as observed by us in morphant Xenopus CNC cells ${ }^{24}$, supporting a more general function of CS/DS in polarized cell migration. Interestingly, deficient CS/ DS synthesis in MC-EDS patients resulted in a reduced and less organized fibronectin assembly in the ECM of cultured dermal fibroblasts ${ }^{5}$, underscoring that a disturbed interaction of CS/DS-PGs and this matrix protein might 
contribute to the pathophysiology of this disorder. The integrin $\alpha 5 \beta 1$ heterodimer ${ }^{27,31}$ and Syndecan $4^{32,33}$ bind to distinct domains of fibronectin and participate in NC cellmatrix adhesion and cell migration. Yet, we did not find CS/DS chains in integrin $\alpha 5 \beta 1$ and Syndecan 4 , and DSepi1 deficiency did not change their expression ${ }^{24}$. Thus, the identity of the CS/DS-PG and the mechanism of how IdoA promotes $\mathrm{CNC}$ cell adhesion and migration are not yet understood.

In whole embryos and CNC cells, DS-epi1 gave rise to CS/DS chains that consisted of isolated IdoA residues surrounded by unmodified glucuronic acid moieties ${ }^{24}$. This result in Xenopus is remarkable since previous studies in the connective tissue of Dse knockout mice ${ }^{12}$ and a human patient with Dse dysfunction ${ }^{4}$ rather suggested a role of DS-epi1 in the formation of IdoA blocks, i.e. stretches of consecutive IdoA. The protein core, tissue type and signaling status are known to influence the number and distribution of IdoA moieties in CS/DS chains ${ }^{6,34}$. The binding to growth factors and ECM components can be critically dependent on even a single IdoA. This was previously demonstrated in vitro for example by the high affinity of the growth factor pleiotrophin to CS/DS oligosaccharide sequences that were extracted from the embryonic pig brain ${ }^{35}$.To our knowledge, the study in the Xenopus embryo is the first to show that isolated IdoA in CS/DS has a function in vivo ${ }^{24}$.

The DS-epi1 knockdown in Xenopus embryos ${ }^{24}$ mimics the craniofacial defect in MC-EDS. Our experimental study suggests that impaired EMT, failure of NC cells to attach to fibronectin and/or cell death as a consequence of anchorage loss might explain the inadequate number of NC cells that migrate to their correct destination. We therefore propose to add MC-EDS to the list of neurocristopathies. Impaired NC development might not only contribute to craniofacial anomalies but explain other congenital malformations of this syndrome. For example, hearing impairment, heart defects (valve abnormalities) and gastrointestinal features (constipation) are frequently encountered in MC-EDS patients ${ }^{3}$ and could be linked to NC defects. Considering the diverse cell types and tissues that NC cells generate ${ }^{36}$, further NC-related anomalies might be uncovered that improve the diagnosis and allow a tailored therapy of this at present intractable disorder.

\section{Acknowlegement}

This work was financially supported by the Swedish Research Council (2009-4951 to E.M.P.), Swedish Childhood Cancer Foundation (PROJ11/101 to E.M.P., NBCNSPDHEL12/012 to N.G.), and Swedish Cancer Foundation (grant 140530 to M.M.).

\section{References}

1. Malfait F, De Paepe A. The Ehlers-Danlos syndrome. Adv Exp Med Biol. 2014; 802: 129-143.
2. Zhang L, Müller T, Baenziger JU, et al. Congenital disorders of glycosylation with emphasis on loss of dermatan-4-sulfotransferase. Prog Mol Biol Transl Sci. 2010; 93: 289-307.

3. Kosho T. CHST14/D4ST1 deficiency: A new form of Ehlers-Danlos syndrome. Pediatr Int. 2016; 58: 88-99.

4. Müller T, Mizumoto S, Suresh I, et al. Loss of dermatan sulfate epimerase (DSE) function results in musculocontractural EhlersDanlos syndrome. Hum Mol Genet. 2013; 22: 3761-3772.

5. Syx D, Damme T, Symoens S, et al. Genetic Heterogeneity and Clinical Variability in Musculocontractural Ehlers-Danlos Syndrome Caused by Impaired Dermatan Sulfate Biosynthesis. Human Mut. 2015; 36: 535-547.

6. Trowbridge JM, Gallo RL. Dermatan sulfate: new functions from an old glycosaminoglycan. Glycobiology. 2002; 12: 117R-125R.

7. Thelin MA, Bartolini B, Axelsson J, etal. Biological functions of iduronic acid in chondroitin/dermatan sulfate. FEBS J. 2013; 280: 2431-2446.

8. Iozzo RV, Schäfer L. Proteoglycan form and function: A comprehensive nomenclature of proteoglycans. Matrix Biol. 2015; 42: 11-55.

9. Quentin E, Gladen A, Rodén L, et al. A genetic defect in the biosynthesis of dermatan sulfate proteoglycan: galactosyltransferase I deficiency in fibroblasts from a patient with a progeroid syndrome. Proc Natl Acad Sci U S A. 1990; 87:1342-1346.

10. Malfait F, Kariminejad A, Van Damme T, et al. Defective initiation of glycosaminoglycan synthesis due to B3GALT6 mutations causes a pleiotropic Ehlers-Danlos-syndrome-like connective tissue disorder. Am J Hum Genet. 2013; 92: 935-945.

11. Nakajima M, Mizumoto S, Miyake N, et al. Mutations in B3GALT6, which encodes a glycosaminoglycan linker region enzyme, cause a spectrum of skeletal and connective tissue disorders. Am J Hum Genet. 2013; 92: 927-934.

12. Maccarana M, Kalamajski S, Kongsgaard M, et al. Dermatan sulfate epimerase 1-deficient mice have reduced content and changed distribution of iduronic acids in dermatan sulfate and an altered collagen structure in skin. Mol Cell Biol. 2009; 29: 5517-5528.

13. Miyake N, Kosho T, Mizumoto S, et al. Loss-of-function mutations of CHST14 in a new type of Ehlers-Danlos syndrome. Hum Mutat. 2010; 31: 966-974.

14. Gustafsson R, Stachtea X, Maccarana M, et al. Dermatan sulfate epimerase 1 deficient mice as a model for human abdominal wall defects. Birth Defects Res A Clin Mol Teratol. 2014; 100: 712-720.

15. Trainor PA, Andrews BT. Facial dysostoses: Etiology, pathogenesis and management. Am J Med Genet C Semin Med Genet. 2013; 163C: 283-294.

16. Mayor R, Theveneau E. The neural crest. Development. 2013; 140: 2247-2251.

17. Simões-Costa M, Bronner ME. Establishing neural crest identity: a gene regulatory recipe. Development. 2015; 142: 242-257.

18. Zhang D, Ighaniyan S, Stathopoulos $L$, et al. The neural crest: a versatile organ system. Birth Defects Res C Embryo Today. 2014; 102: 275-298.

19. Perris R, Perissinotto D. Role of the extracellular matrix during neural crest cell migration. Mech Dev. 2000; 95, 3-21.

20. Perissinotto D, Iacopetti P, Bellina I, et al. Avian neural crest cell migration is diversely regulated by the two major hyaluronan-binding proteoglycans PG-M/versican and aggrecan. Development. 2000; 127: 2823-2842.

21. Dutt S, Kléber M, Matasci M, et al. Versican V0 and V1 guide migratory neural crest cells. J Biol Chem. 2006; 281: 12123-12131.

22. Casini P, Ori M, Avenoso A, et al. Identification and gene expression of versican during early development of Xenopus. Int J Dev Biol. 2008; 52: 993-998. 
23. Szabó A, Melchionda M, Nastasi G, et al. In vivo confinement promotes collective migration of neural crest cells. J Cell Biol. 2016; 213: 543-555.

24. Gouignard N, Maccarana M, Strate I, et al. Musculocontractural Ehlers-Danlos syndrome and neurocristopathies: dermatan sulfate is required for Xenopus neural crest cells to migrate and adhere to fibronectin. Dis Model Mech. 2016; 9: 607-620.

25. Pacheco B, Maccarana M, Goodlett DR, et al. Identification of the active site of DS-epimerase 1 and requirement of $\mathrm{N}$-glycosylation for enzyme function. J Biol Chem. 2009; 284: 1741-1747.

26. Davidson LA, Keller R, DeSimone DW. Assembly and remodeling of the fibrillar fibronectin extracellular matrix during gastrulation and neurulation in Xenopus laevis. Dev Dyn. 2004; 231: 888-895.

27. Alfandari D, Cousin H, Gaultier A, et al. Integrin $\alpha 5 \beta 1$ supports the migration of Xenopus cranial neural crest on fibronectin. Dev Biol. 2003; 260: 449-464.

28. Saito A, Munakata H. Analysis of plasma proteins that bind to glycosaminoglycans. Biochim Biophys Acta. 2007; 1770: 241-246.

29. Thelin MA, Svensson KJ, Shi X, et al. Dermatan sulfate is involved in the tumorigenic properties of esophagus squamous cell carcinoma. Cancer Res. 2012; 72: 1943-1952.

30. Bartolini B, Thelin MA, Svensson L, et al. Iduronic acid in chondroitin/ dermatan sulfate affects directional migration of aortic smooth muscle cells. PloS one. 2013; 8: e66704.
31. Franco CR, Trindade ES, Rocha HA, et al. Glycosaminoglycan chains from alpha5beta1 integrin are involved in fibronectin-dependent cell migration. Biochem Cell Biol. 2009; 87: 677-686.

32. Woods A, Longley RL, Tumova S, et al. Syndecan-4 binding to the high affinity heparin-binding domain of fibronectin drives focal adhesion formation in fibroblasts. Arch Biochem Biophysics. 2000; 374: $66-72$.

33. Matthews HK, Marchant L, Carmona-Fontaine C, et al. Directional migration of neural crest cells in vivo is regulated by Syndecan-4/ Rac1 and non-canonical Wnt signaling/RhoA. Development. 2008; 135: $1771-1780$

34. Tiedemann K, Olander B, Eklund E, et al. Regulation of the chondroitin/ dermatan fine structure by transforming growth factor-beta1 through effects on polymer-modifying enzymes. Glycobiology. 2005; 15: 1277 1285

35. Bao X, Muramatsu T, Sugahara K. Demonstration of the pleiotrophinbinding oligosaccharide sequences isolated from chondroitin sulfate/ dermatan sulfate hybrid chains of embryonic pig brains. J Biol Chem. 2005; 280:35318-35328.

36. Dupin E, Le Douarin NM. The neural crest, a multifaceted structure of the vertebrates. Birth Defects Res C Embryo Today. 2014; 102: 187 209. 